\title{
A Randomized Feasibility Trial Comparing Surveillance Regimens for Patients with Low and Low-Intermediate Risk Non-Muscle Invasive Bladder Cancer
}

\author{
Ryan M. Reyes ${ }^{\mathrm{a}}$, Emily Rios ${ }^{\mathrm{b}}$, Shane Barney ${ }^{\mathrm{b}}$, Cory M. Hugen ${ }^{\mathrm{b}}$, Joel E. Michalek ${ }^{\mathrm{c}}$, Yair Lotan ${ }^{\mathrm{d}}$, \\ Edward M. Messing and Robert S. Svatek ${ }^{\mathrm{a}, \mathrm{b}, *}$ \\ ${ }^{a}$ Mays Cancer Center, University of Texas Health San Antonio, San Antonio, TX, USA \\ ${ }^{\mathrm{b}}$ Department of Urology, University of Texas Health San Antonio, San Antonio, TX, USA \\ ${ }^{\mathrm{c}}$ Department of Epidemiology and Biostatistics, University of Texas Health San Antonio, San Antonio, TX, USA \\ ${ }^{\mathrm{d}}$ Department of Urology, University of Texas Southwestern Medical Center, Dallas, TX, USA \\ ${ }^{\mathrm{e}}$ Department of Urology, University of Rochester, Rochester, NY, USA
}

Received 11 December 2020

Accepted 15 May 2021

Pre-press 3 June 2021

Published 31 August 2021

\begin{abstract}
.
BACKGROUND: Surveillance regimens for non-muscle invasive bladder cancer (NMIBC) are disparate and controlled trials could inform guidelines. The feasibility of randomizing patients to variable frequency surveillance is unknown.

OBJECTIVES: To determine patient willingness to randomization to high frequency (HF) versus low frequency (LF) surveillance regimen for NMIBC and compare patient comfort and healthcare costs across regimens.

METHODS: A non-blinded, two-arm, randomized-controlled study of patients with low or low-intermediate risk NMIBC was conducted at two institutions where patients were offered randomization to HF vs. LF surveillance following initial tumor resection. The HF group underwent cystoscopy every three months for 2 years, then every 6 months for 2 years, then annually. The LF group underwent cystoscopy at 9 months following the 3-month cystoscopy, then annually. Assuming $75 \%$ of patients approached would agree to enrollment, a sample size of $n=35$ patients per arm provided a one-sided $95 \%$ exact Clopper-Pearson confidence lower-limit of $60 \%$.

RESULTS: Of 70 patients approached, $45(64.3 \%)$ agreed to participate and 25 (35.7\%) declined enrollment due to preference for HF. Twelve biopsies were performed, including 4 (19\%) of 21 patients in the HF group and 8 (33.3\%) of 24 patients in the LF group. Disease recurrence (low grade Ta) was observed in $3(14.3 \%)$ and 5 (20.8\%) patients in the HF and LF groups, respectively. No patients experienced high grade recurrence or progression. Both groups had similar patient-reported procedure-related discomfort and quality of life measures over time. Patient out-of-pocket cost and healthcare systems costs were $\$ 383.80$ more per patient annually in the HF group.
\end{abstract}

\footnotetext{
*Correspondence to: Robert S. Svatek, MD, MSCI, Department of Urology, University of Texas Health San Antonio, Mays Cancer
} 
CONCLUSIONS: Randomization to variable frequency surveillance is challenging as over a third of patients declined participation. However, these data provide important preliminary insights into the potential effects of surveillance frequency on oncologic and economic outcomes in patients with low and low-intermediate risk bladder cancer.

Keywords: Bladder cancer, surveillance, recurrence, quality of life

\section{ABBREVIATIONS KEY}

\begin{tabular}{|c|c|}
\hline $\begin{array}{l}\text { AUA } \\
\text { CIS }\end{array}$ & $\begin{array}{l}\text { American Urologic Association } \\
\text { carcinoma } i n \text {-situ }\end{array}$ \\
\hline EAU & European Association of Urology \\
\hline FACT-BL & $\begin{array}{l}\text { Functional Assessment of Cancer } \\
\text { Therapy-Bladder }\end{array}$ \\
\hline $\mathrm{HF}$ & High frequency \\
\hline LF & Low frequency \\
\hline NMIBC & Non-muscle invasive bladder cancer \\
\hline TURBT & $\begin{array}{l}\text { Transurethral resection of bladder } \\
\text { tumor }\end{array}$ \\
\hline
\end{tabular}

\section{INTRODUCTION}

Bladder cancer is the 6th most common cancer in the U.S., with an expected 81,400 newly diagnosed cases and 17,980 deaths in 2021 [1]. Of these new cases, approximately $85 \%$ present as non-muscle invasive bladder cancer (NMIBC). Transurethral resection of bladder tumor (TURBT) is the primary diagnostic procedure for new and recurrent bladder tumors and can be both diagnostic and therapeutic. Due to the high likelihood of disease recurrence after initial treatment and the possibility of disease progression, NMIBC patients require longterm endoscopic monitoring. The need for long-term surveillance contributes to increased cost for the care of bladder cancer, contributing significant expense to health care systems [2]. Given that the vast majority of office-based cystoscopies are negative for recurrence, strategies to reduce the number of cystoscopies are needed. These include (1) improving diagnostic accuracy of cystoscopy by utilizing imaging enhancement such as narrow-band imaging [3] and use of bluelight cystoscopy [4], (2) replacement of cystoscopy with urine-based biomarkers $[5,6]$ or bladder imaging [7], and (3) decreased frequency of cystoscopic surveillance $[8,9]$.

Guidelines for surveillance monitoring have evolved. Following the risk-adapted strategy developed by the European Association of Urology (EAU) [10], in 2016, the American Urological Association (AUA) guidelines recommend a less frequent surveillance regimen for low risk patients, defined as six to nine months after first surveillance cystoscopy, and then annually for five years [11]. These guidelines were largely based on expert opinion without prospective clinical trial data to guide such decisions. The periodicity of surveillance cystoscopy could influence the natural biology of the disease if time to detection is altered, necessitating prospective randomized-controlled studies to evaluate patient outcomes. In the U.S., randomization to alternative frequency surveillance modalities is challenging as patients and/or their providers may not hold sufficient equipoise to agree to randomization. Here, we conducted a feasibility trial to address variable frequency surveillance evaluations for patients with low and low-intermediate risk NMIBC. Patients were randomized to a more versus less frequent cystoscopic surveillance regimen based on prior and current AUA and EAU guidelines. The primary objective was to determine feasibility and to facilitate phase III trial development. Secondary objectives were to estimate patient burden in terms of disease relapse, additional procedures, patient satisfaction or dissatisfaction, pain, and cost associated with the surveillance strategy.

\section{MATERIALS AND METHODS}

A non-blinded, two-arm, randomized-controlled study was conducted following local institutional review board approval (HSC\# 2013-0177H) and the full study protocol is included in the Supplementary Data. We used 2008 EAU guidelines for risk of disease recurrence/progression which were assigned based on a scoring system that accounts for the number of tumors, patient's prior recurrence rate, presence of carcinoma in-situ (CIS), tumor size, grade, and stage [10]. Recurrence scores and corresponding risk assessment were as follows: 0, low risk; 1-4, low-intermediate risk; 5-9 intermediate-high risk; $10-17$ high risk. Eligibility criteria: All patients had low risk or low-intermediate risk for disease recurrence and/or progression. Patients had at least one negative surveillance cystoscopy at 3 months 

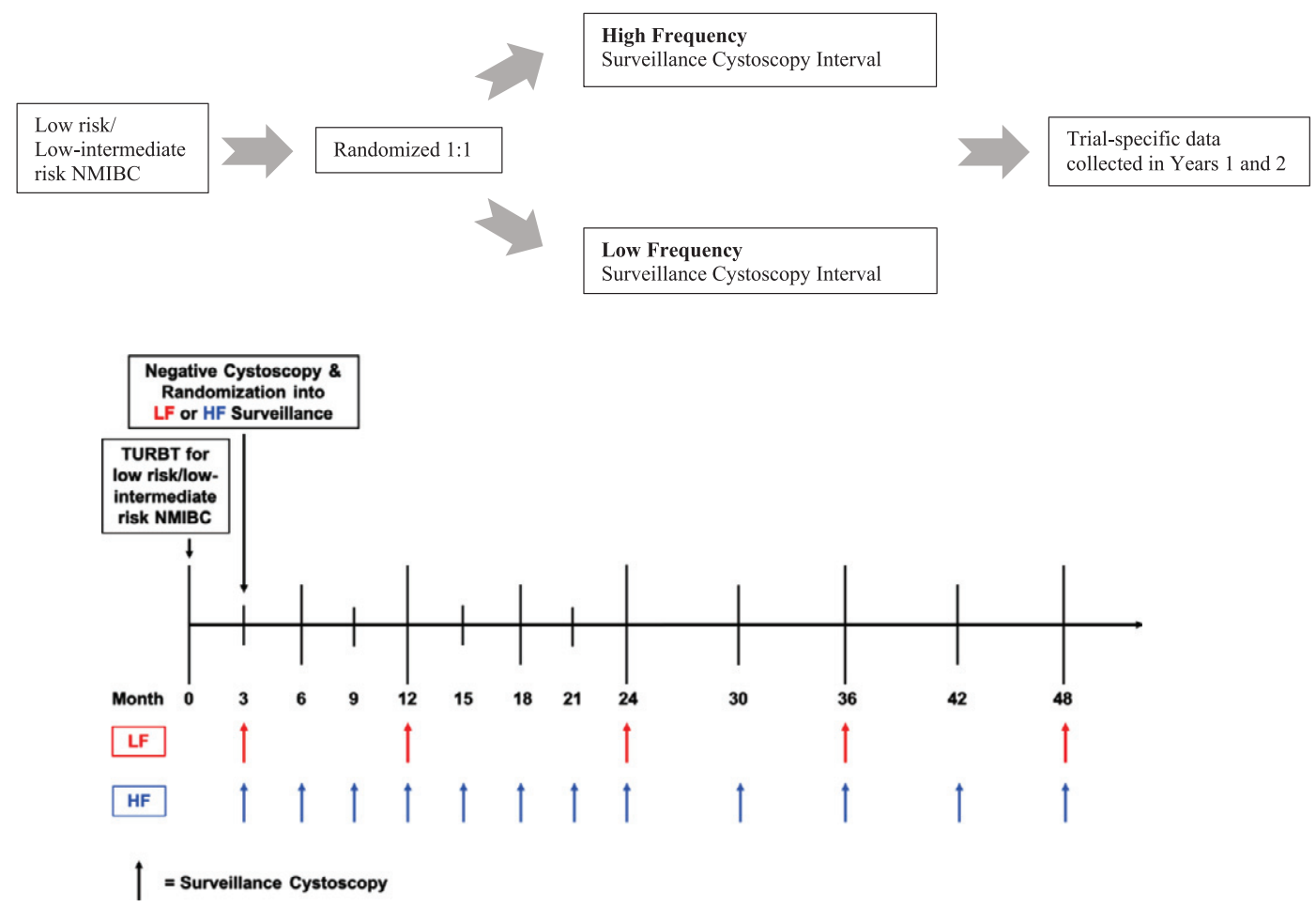

Fig. 1. Trial schema and timeline of surveillance cystoscopies across low and high frequency surveillance groups.

following the most recent biopsy. The diagnosis had to be less than 48 months prior to enrollment. Patients were excluded if they had a history of muscle invasive bladder cancer, CIS, or variant histology (e.g., micropapillary, nested variant, non-urothelial cell carcinoma elements). All patients were over 18 years old and gave informed consent. The study was registered with clinicaltrials.gov (NCT02298998) and occurred at the University of Texas Health Science Center San Antonio and the University of Texas Southwestern Medical Center from April 2013 to May 2020. However, the trial was not actively accruing patients during this entire time period.

Patients were randomized to one of two surveillance regimens, termed high frequency (HF) and low frequency (LF) (Fig. 1). If the participant was randomized to the HF arm, surveillance cystoscopy was performed every 3 months for 2 years, then every 6 months for the next 2 years, and then annually thereafter. If the participant was randomized to the LF arm, surveillance cystoscopy was performed 9 months after the negative 3-month cystoscopy, then annually for 5 years. All potentially recurrent lesions underwent biopsy for pathologic assessment. The utilization of cytology was at the treating urologist's discretion as per their usual standard of care and disease recurrence was confirmed with pathologic assessment. Study duration was 2 years from the time of study enrollment.

Participants were administered self-reported questionnaires after each cystoscopy visit which aimed to capture their overall attitudes and satisfaction/dissatisfaction regarding the follow-up regimen for their bladder cancer. The patient satisfaction questionnaire (Appendix) focused on the discomfort and bother associated with the procedure. The patientreported cost assessment questionnaire (Appendix) asked questions to determine amount of time off work, transportation, travel time, and out of pocket costs associated with each visit. The Functional Assessment of Cancer Therapy-Bladder (FACT-BL) questionnaire included a Likert scale (0-4) aimed at assessing patient attitudes regarding physical, emotional, social/family, and functional well-being, as well as other concerns.

The personal cost of each visit was calculated by adding patients' self-reported expenses. The cost of the appointment, which was universally reported as the co-pay, was included. In addition, all patients recorded the total mileage traveled for the appointment as well as any other additional expenses. In cases where patients failed to provide precise 


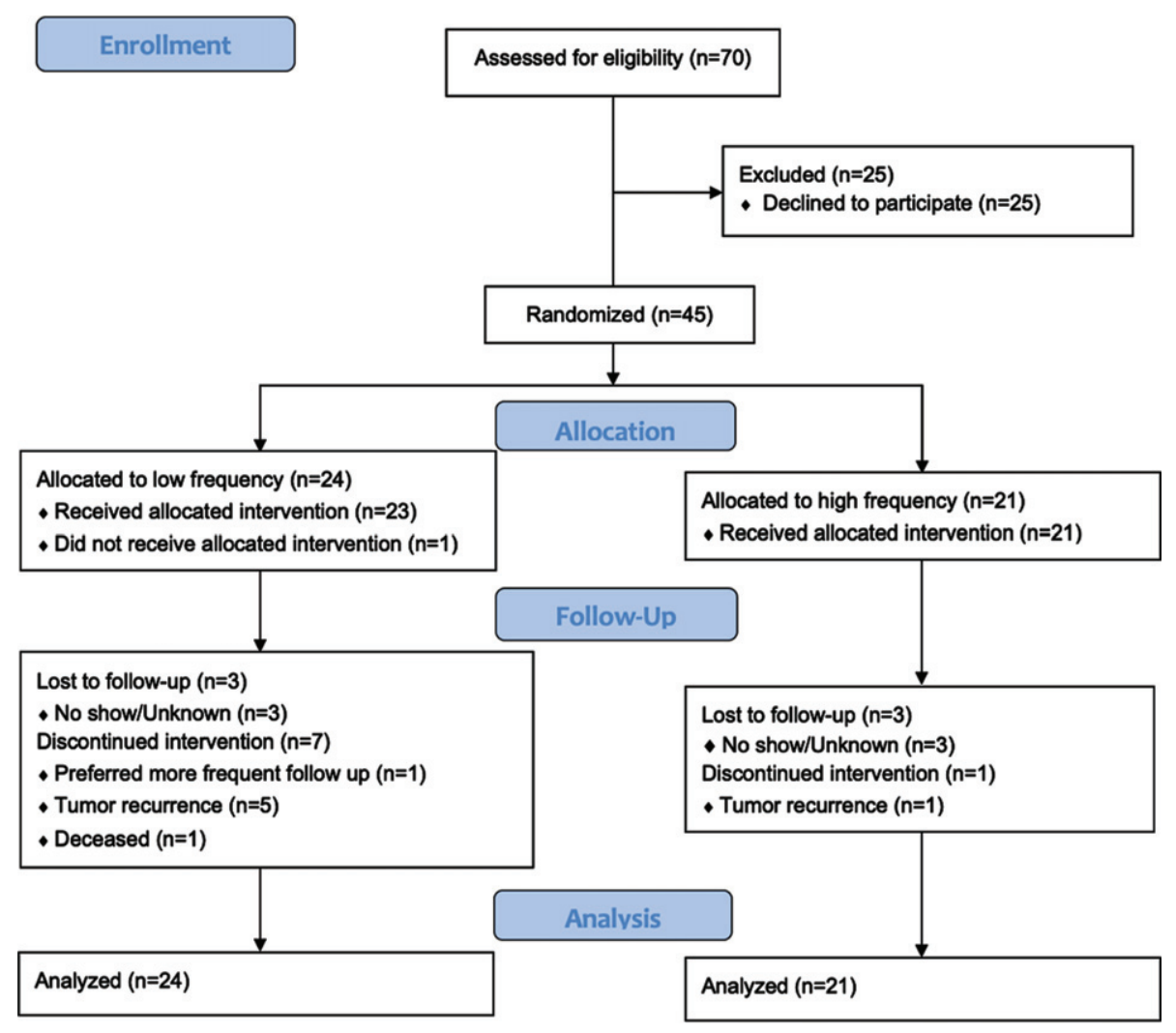

Fig. 2. CONSORT flow diagram. Source: http://www.consort-statement.org/consort-statement/flow-diagram.

monetary value of expense, we estimated the expense using standardized average costs (e.g., one hotel night $\$ 100$, meal $\$ 8$, and parking \$5). To convert driven mileage into a numbered cost, we used the average reported regional cost per gallon for the years of the study (\$2.36/gallon) and average national fuel economy for those years $(23.6 \mathrm{miles} / \mathrm{gallon}$; estimated $\$ 0.10 / \mathrm{mile})$. Money lost for time off work was not included in the questionnaire. Although the cost of cystoscopy changed over the time of enrollment and surveillance, the 2020 Medicare reimbursement for in office cystoscopy was $\$ 216.18$ and was used in our calculation of total cost for cystoscopy.

The primary objective of the study was to determine the recruitment rate for a randomized surveillance trial for patients with NMIBC. The sample size for this pilot study was calculated as follows: Letting $p$ denote the proportion of patients who enrolled from among the patients approached to participate, our goal was to choose a sample size to produce an informative lower-limit (one-sided) confidence interval for $\mathrm{p}$. Assuming $p=0.75$, a sample size of 35 per arm produces a one-sided 95\% exact Clopper-Pearson lower-limit confidence interval with a distance from the sample proportion to the lower limit is equal to 0.15 . With this assumption, the expected lower confidence limit will be 0.602 [PASS, NCSS, Kaysville, Utah, 2011]. Due to the lack of prior data, we are unable to hypothesize the value of $p$ specific to each arm and so chose to follow a balanced design with $n=35$ subjects per arm and a total sample size of $n=70$. The number of patients screened, the number of screen failures by reason, the number randomized to each arm, and the number of patients who subsequently withdrew were tabulated (Fig. 2). Tabular summaries of baseline characteristics, and numeric outcomes pertaining to all primary and secondary end points were summarized by study arm (Table 1). Intervention and control arms were contrasted on binary and categorical outcomes with Fisher's Exact Test and on continuously distributed outcomes with $t$-tests or Wilcoxon tests as appropriate. All statistical testing was conducted with 2 -sided tests at a significance level of 5\%. Corrections for multiple testing were not applied. 


\section{RESULTS}

A total of 70 eligible patients were approached and $45(64.3 \%)$ agreed to participation. Of the 45 participants, $21(46.7 \%)$ were randomized to $\mathrm{HF}$ and $24(53.3 \%)$ were randomized to LF. The groups were similar across gender, race, ethnicity, bladder cancer family history, smoking status, risk to recurrence/progression, and prior intravesical treatment history (Table 1). Of the HF group, low and lowintermediate risk disease was present in $66.7 \%$ and $33.3 \%$ of patients, respectively. Of the LF group, low and low-intermediate risk disease was present in $45.8 \%$ and $54.2 \%$ respectively. Two patients from the low-intermediate risk group had high grade disease. All other enrolled patients were low grade Ta.

Table 1

Patient Demographics

\begin{tabular}{|c|c|c|}
\hline & $\mathrm{HF}(n=21)$ & $\mathrm{LF}(n=24)$ \\
\hline Mean age & 70.2 & 68.4 \\
\hline \multicolumn{3}{|l|}{ Gender } \\
\hline Male & $17(81.0 \%)$ & $20(83.3 \%)$ \\
\hline Female & $4(19.0 \%)$ & $4(16.7 \%)$ \\
\hline \multicolumn{3}{|l|}{ Race } \\
\hline White & $18(85.7 \%)$ & $22(91.7 \%)$ \\
\hline Black & $3(14.3 \%)$ & $2(8.3 \%)$ \\
\hline \multicolumn{3}{|l|}{ Ethnicity } \\
\hline Non-Hispanic & $15(71.4 \%)$ & $17(70.8 \%)$ \\
\hline Hispanic & $6(28.6 \%)$ & $7(29.2 \%)$ \\
\hline \multicolumn{3}{|l|}{ Insurance } \\
\hline Private & 8 & 10 \\
\hline Medicare & 14 & 14 \\
\hline Medicaid & 1 & 2 \\
\hline State/County & 0 & 0 \\
\hline Self-Pay & 0 & 0 \\
\hline Other & 6 & 9 \\
\hline \multicolumn{3}{|l|}{ Smoking Status } \\
\hline Current & $4(19.0 \%)$ & $2(8.3 \%)$ \\
\hline Previous & $16(76.2 \%)$ & $14(58.3 \%)$ \\
\hline Never & $1(4.8 \%)$ & $8(33.3 \%)$ \\
\hline \multicolumn{3}{|l|}{ Bladder Cancer Family History } \\
\hline Positive & $4(9.0 \%)$ & $6(25.0 \%)$ \\
\hline Negative & $17(81.0 \%)$ & $18(75.0 \%)$ \\
\hline \multicolumn{3}{|l|}{ Risk } \\
\hline Low & $14(66.7 \%)$ & $11(45.8 \%)$ \\
\hline Low-Intermediate & $7(33.3 \%)$ & $13(54.2 \%)$ \\
\hline History of Prior Intravesical Therapy & $8(38.1 \%)$ & $6(25.0 \%)$ \\
\hline
\end{tabular}

Of the 45 patients who initially agreed to participate, $14(31 \%)$ patients subsequently withdrew for reasons that included study duration, burden, and randomization group (Fig. 2).

A total of 12 patients underwent biopsy for a suspicious lesion seen on cystoscopy during the study period, including $4(19.0 \%)$ in the $\mathrm{HF}$ and 8 (33.3\%) in the LF groups (Table 2). Disease recurrence was defined pathologically and observed in $3(14.3 \%)$ patients in the HF group and $5(20.8 \%)$ patients in the LF group $(P=0.71)$. All recurrences were less than $3 \mathrm{~cm}$, low grade Ta, and no patients progressed. Average time to recurrence in patients experiencing disease relapse was 10.7 months and 9.6 months for the HF and LF groups respectively, and 4 (16.0\%) patients were low risk and $4(20.0 \%)$ were lowintermediate risk at time of study entry (Table 2). Of the low-intermediate risk recurrences, none included the two patients with original pathology of high grade. A subgroup analysis was performed for the patients enrolled at time of their first surveillance cystoscopy after diagnosis which confirmed no statistical difference between the groups in either number of biopsies or recurrences. The median follow-up was 21.9 months for the HF group and 10.9 months for the LF group. When subgroup analysis was performed removing those withdrawn due to a recurrence, median follow up from the first negative cystoscopy was 22.3 and 16.3 months for HF and LF groups, respectively.

Patient-reported quality of life scores were examined over time for the HF and LF groups. The mean (SD) percent change from baseline between the total FACT-BL scores from enrollment compared to those 2 years later at time of study termination was $4.06 \%$ increased from baseline for the HF group and $-1.38 \%$ from baseline for the LF group $(P=0.65)$. The mean (SD) percent change from baseline between the discomfort scores on the satisfaction questionnaire were $-28.2 \%$ in the HF group and $10.1 \%$ in the LF group $(P=0.62)$. Graphical representation of FACT$\mathrm{BL}$ scores show a gradual decline in physical and emotional scores over the duration of the study but not significant differences in the slope of the curves between HF and LF groups (Fig. 3).

Table 2

Biopsy Characteristics

\begin{tabular}{lcccccc}
\hline & HF $(n=21)$ & LF $(n=24)$ & $P$-value & $\begin{array}{c}\text { Low Risk } \\
(N=25)\end{array}$ & $\begin{array}{c}\text { Low-Intermediate } \\
(N=20)\end{array}$ & $P$-value \\
\hline No. biopsies (\%) & $4(19)$ & $8(33)$ & 0.33 & $6(24)$ & $6(30)$ & 0.74 \\
No. of recurrences (\%) & $3(14)$ & $5(21)$ & 0.71 & $4(16)$ & $4(20)$ & 1.00 \\
\hline
\end{tabular}


A

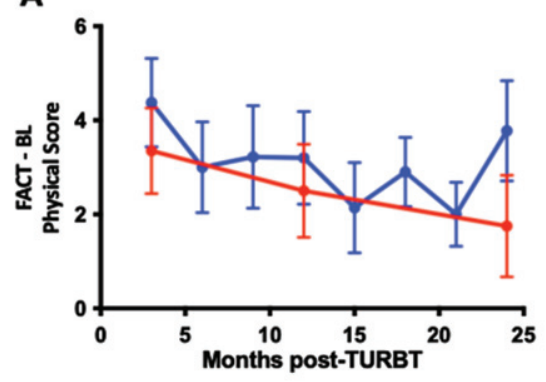

C

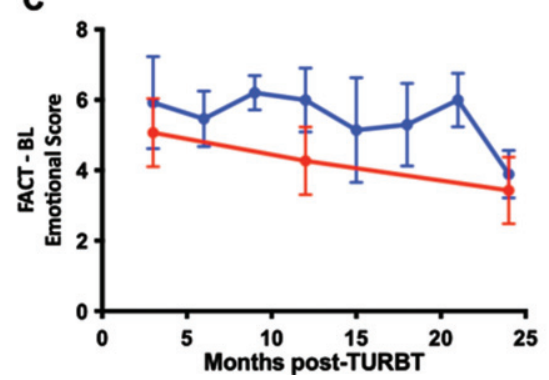

B
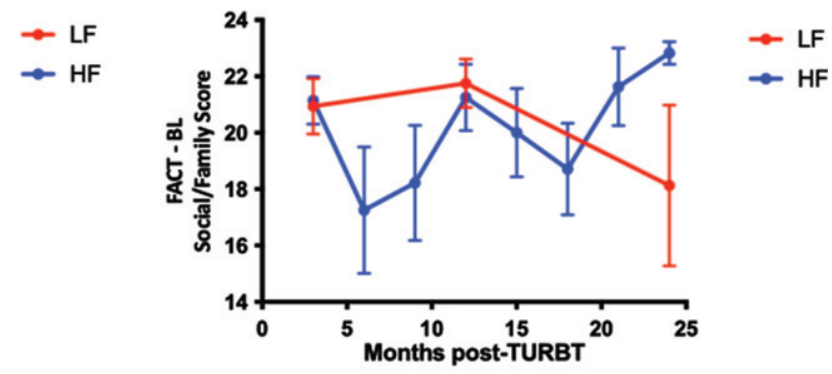

D
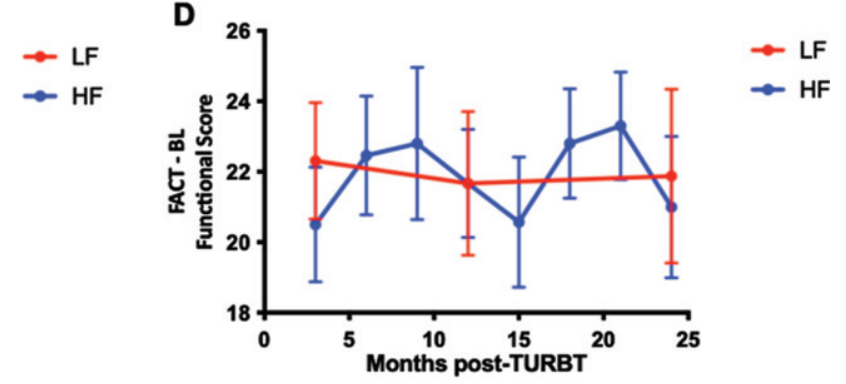

Fig. 3. Patient-reported outcomes are similar for LF and HF surveillance regimens. Functional assessment of cancer therapy (FACT) Bladder (BL) patient reported quality of life questionnaire scores assessing physical (A), social/family (B), emotional (C), and functional (D) well-being over time. Mean \pm SEM.

A

Patient Out-of-Pocket Expenses

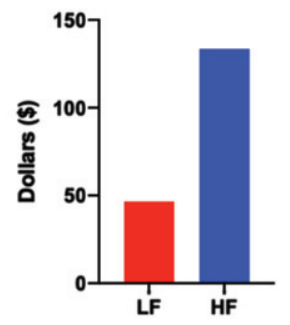

B

Healthcare System Cystoscopy Cost

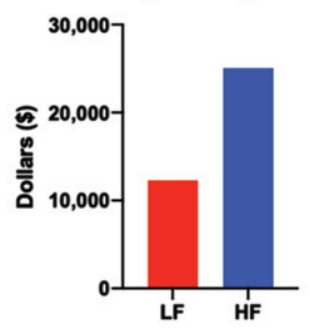

C Annual Economic Cost Per Patient

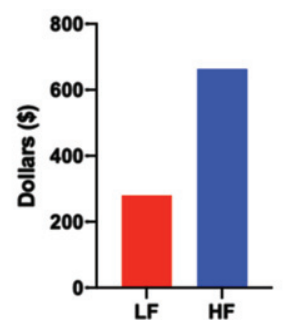

Fig. 4. Cystoscopy surveillance regimen impacts healthcare system expenses more than patient out-of-pocket costs. (A) Average patient out-of-pocket costs for the duration of the surveillance regimen. (B) Average healthcare system costs based on Medicare reimbursement per cystoscopy and cystoscopy frequency in each regimen. (C) Estimated annual economic cost calculated by adding yearly patient out-of-pocket costs and cystoscopy regimen reimbursement costs to healthcare system.

In total, patient-reported out-of-pocket expenses in the HF group were $\$ 2,805.00$ for 116 cystoscopies $(n=21$ patients), averaging $\$ 133.57$ per patient. In the LF group, the total out-of-pocket cost was $\$ 1120.30$ for 57 cystoscopies ( $n=24$ patients), averaging $\$ 46.68$ per patient with a total cost difference per patient of $\$ 86.89$ (Fig. 4A). Differences in healthcare cost were calculated by multiplying the number of cystoscopies from each regimen by the current Medicare reimbursement rate per cystoscopy (Fig. 4B). The total annual economic cost was cal- culated by adding the healthcare system cost of cystoscopy and patient-reported out of pocket costs, revealing a more dramatic cost difference of $\$ 663.85$ and \$280.05 annually per patient in the HF and LF regimen, respectively (Fig. 4C). At approximately 80,000 new patients diagnosed with bladder cancer annually in the U.S. and $30 \%$ of them having low or low-intermediate risk disease, this translates into a cost difference between HF versus LF surveillance of more than 9 million dollars annually. Additionally, the potential economic impact of a reduced 
surveillance regimen is even greater given that an estimated 700,000 people in the U.S. have bladder cancer [12], with approximately $85 \%$ of all patients having NMIBC and likely receiving some form of surveillance.

\section{DISCUSSION}

Surveillance regimens in bladder cancer utilize considerable resources. Existing guidelines for surveillance in NMIBC are largely based on expert opinion. Higher levels of evidence, including prospective comparative trials are needed to support these surveillance guidelines. However, patient willingness to participate in randomized trials where patients are delegated to variable surveillance regimens is not clear. This trial compared two surveillance protocols that differed drastically in the frequency of cystoscopy for patients with low and lowintermediate risk NMIBC. While the majority of patients approached agreed to participate, the relatively high rate of patient decline or withdrawal indicates significant barriers to successful completion of a phase III trial, with many patients declining randomization based on preference for more frequent cystoscopy. While our study was not designed to assess a difference in oncologic outcomes, no difference was observed between the HF and LF groups in terms bladder biopsies or disease recurrence. The fact that no patients progressed supports this group of patients as an appropriate population to study surveillance frequency without untoward outcome. Patient-reported quality of life assessments were similar across groups and, as expected, the costs were significantly lower in the LF group compared to the HF group. Collectively, these findings support the current AUA surveillance guidelines for low risk NMIBC, which recommend lower frequency cystoscopies compared to patients with high risk NMIBC.

Out of pocket cost in the setting of NMIBC surveillance is poorly reported. In 2004, 6370 respondents with a diagnosis of any cancer not being treated reported an annual out-of-pocket cost of $\$ 1450$ [15]. To illustrate the financial burden further, $27 \%$ of lowincome patient's earnings went to treatment of cancer. Bladder cancer has had the highest cost for treatment per patient in the U.S. of all cancers, which ranged from $\$ 96,000$ to $\$ 187,000$ [2]. The cost of diagnosis and follow up for NMIBC, not including treatment, has been estimated to be $\$ 4000$ per patient over a 5-year period. Consequently, we predicted that our measured out-of-pocket cost to the patient would also be a sizeable financial burden. Unexpectedly, our results didn't confirm this. It is unclear why this may be, but as previously stated, the calculated cost didn't take into account money lost from being away from work. In addition, 23 of the patients were recruited from the Veterans Affairs medical system where there is often transportation provided, no co-pay, and other ancillary services to decrease out-of-pocket expenses for the patient. Lastly, costs could have been underreported by patients.

One prior report compared two different surveillance cystoscopy regimens for patients with papillary NMIBC and randomized 97 patients [8]. Similar to our study, there was no difference between the risk of recurrence or progression between groups. In addition, the number of visits was reduced by $37.5 \%$ in the lower frequency group which is less than the $50.1 \%$ reduction in the number of visits observed in our trial.

Several patients randomized to the LF group withdrew from our study, due to patient preference for a higher frequency regimen. Future studies could combine reduced frequency surveillance with cost effective measures aimed at providing patients additional reassurance that tumors have not recurred, such as urine-based biomarker testing occurring between surveillance cystoscopies. However, prior studies have shown minimal patient willingness to reduce cystoscopy frequency in lieu of a urine-based recurrence monitoring without a high degree of certainty of urine-based testing sensitivity for tumor detection $[13,14]$. Additionally, a nuanced discussion with low and low-intermediate risk patients on the incidence of disease recurrence $(\sim 15 \%)$ and lack of association with disease progression $(<1 \%)$ [16] may lead to additional comfort with reduced surveillance regimens.

The are several methods that can be improved during conduction of a subsequent phase trial. One important metric that was not captured was fear of recurrence, a recently identified outcome in psychosocial cancer research and clinical care [17]. Fear of recurrence imposes a significant burden in patients following definitive treatment and during surveillance of cancer and, therefore, could influence quality of life and cancer outcomes indirectly by impacting surveillance compliance and willingness to participate in trials. Although FACT-BL scores in the emotional domain were similarly low in both HF and LF groups, future studies should more closely investigate disease-related anxiety and fear of recurrence. Also, our study did not directly address 
patients' attitudes and desires regarding frequency of surveillance, which would be an important item for further research. Our cost questionnaire recorded easily quantifiable information such as travel distance, but a more comprehensive cost capturing system is recommended in subsequent studies. Finally, this trial addressed extremes of cystoscopy frequency and the patient willingness for participation may be improved with less drastic differences in regimens between treatment groups. Despite these limitations, our study provides critical data needed to develop subsequent trials and suggests that patient quality of life and satisfaction are not substantially different across variable frequency surveillance regimens.

In summary, accrual to a randomized trial comparing variable frequency surveillance regimens is possible but a significant number of patients declined to participate or withdrew after randomization. Frequent surveillance cystoscopy is tolerated by patients and does not result in substantial increase in outof-pocket costs for patients, supporting use of high frequency surveillance from the patient's perspective. However, similar biopsy, recurrence, and progression rates between low and high frequency surveillance regimens support the use of lower frequency cystoscopies for patients with low and low-intermediate risk disease from a societal perspective.

\section{ACKNOWLEDGMENTS}

The authors thank the patients and clinical care staff that contributed to this trial.

\section{FUNDING}

RM Reyes - NIH T32GM113896, NIH/NCATS TL1 TR002647, NIA T32 AG 021890. RS Svatek 8KL2 TR000118, K23, the Mays Family Cancer Center at University of Texas Health San Antonio (P30 CA054174), Roger L. And Laura D. Zeller Charitable Foundation Chair in Urologic Cancer, CDMRP CA170270/P1P2, the Max \& Minnie Tomerlin Voelcker Fund.

\section{AUTHOR CONTRIBUTIONS}

RM Reyes: interpretation or analysis of data, writing the article. E Rios: performance of work. $S$ Barney: performance of work, interpretation or analysis of data, writing the article. CM Hugen: performance of work. JE Michalek: performance of work. Y Lotan: performance of work. EM Messing: performance of work. RS Svatek: conception, performance of work, interpretation or analysis of data, writing the article.

\section{CONFLICT OF INTEREST}

RMR, ER, SB, CMH, JEM, EMM declare no potential conflicts of interest. YL serves as a consultant for C2I genomics, Photocure, Astra-Zeneca, Merck, Fergene, Abbvie, Cleveland Diagnostics, Bristol Myers Squibb, Nucleix, Ambu, Seattle Genetics, Hitachi, and Ferring Research, has current research relationships with Cepheid, Pacific Edge, MDxHealth, GenomeDX Biosciences, and Storz, and holds leadership positions with Vessi Medical and CAPs Medical. RSS serves as a consultant for Cold Genesys, Ferring Pharmaceuticals, FKD Therapies, Japanese BCG Laboratories, and Nucleix. None of the above-mentioned relationships are directly relevant to the studies described in this manuscript.

\section{SUPPLEMENTARY MATERIAL}

The supplementary material is available in the electronic version of this article: https://dx.doi.org/ 10.3233/BLC-201535.

\section{REFERENCES}

[1] Cancer Stat Facts: Bladder Cancer [https://seer.cancer.gov/ statfacts/html/urinb.html]

[2] Botteman MF, Pashos CL, Redaelli A, Laskin B, Hauser $\mathrm{R}$ : The health economics of bladder cancer: a comprehensive review of the published literature. Pharmacoeconomics. 2003;21(18):1315-30.

[3] Naselli A, Introini C, Timossi L, Spina B, Fontana V, Pezzi R, Germinale F, Bertolotto F, Puppo P: A randomized prospective trial to assess the impact of transurethral resection in narrow band imaging modality on non-muscleinvasive bladder cancer recurrence. Eur Urol. 2012;61(5): 908-13.

[4] Daneshmand S, Patel S, Lotan Y, Pohar K, Trabulsi E, Woods M, Downs T, Huang W, Jones J, O’Donnell M, et al. Efficacy and Safety of Blue Light Flexible Cystoscopy with Hexaminolevulinate in the Surveillance of Bladder Cancer: A Phase III, Comparative, Multicenter Study. J Urol. 2018;199(5):1158-65.

[5] Han M, Schoenberg MP: The use of molecular diagnostics in bladder cancer. Urol Oncol. 2000;5(3):87-92.

[6] Sayyid RK, Sayyid AK, Klaassen Z, Hersey K, Goldberg H, Perlis N, Ahmad A, Leao R, Chandrasekar T, Fadaak K et al: Replacing surveillance cystoscopy with urinary biomarkers in followup of patients with non-muscle-invasive bladder cancer: Patients' and urologic oncologists' perspectives. Can Urol Assoc J. 2018;12(5):E210-E218. 
[7] Kolodziej A, Krajewski W, Matuszewski M, Tupikowski K. Review of current optical diagnostic techniques for non-muscle-invasive bladder cancer. Cent European J Urol. 2016;69(2):150-6.

[8] Olsen LH, Genster HG: Prolonging follow-up intervals for non-invasive bladder tumors: a randomized controlled trial. Scand J Urol Nephrol Suppl. 1995;172:33-6.

[9] Rezaee ME, Lynch KE, Li Z, MacKenzie TA, Seigne JD, Robertson DJ, Sirovich B, Goodney PP, Schroeck FR: The impact of low- versus high-intensity surveillance cystoscopy on surgical care and cancer outcomes in patients with high-risk non-muscle-invasive bladder cancer (NMIBC). PLoS One. 2020;15(3):e0230417.

[10] Babjuk M, Oosterlinck W, Sylvester R, Kaasinen E, Bohle A, Palou-Redorta J: EAU Guidelines on Non-MuscleInvasive Urothelial Carcinoma of the Bladder. Eur Urol. 2008;54(2):303-14.

[11] Chang SS, Boorjian SA, Chou R, Clark PE, Daneshmand S, Konety BR, Pruthi R, Quale DZ, Ritch CR, Seigne JD, et al. Diagnosis and Treatment of Non-Muscle Invasive Bladder Cancer: AUA/SUO Guideline. J Urol. 2016;196(4):1021-9.

[12] Howlader N NA, Krapcho M, Miller D, Bishop K, Kosary CL, Yu M, Ruhl J, Tatalovich Z, Mariotto A, Lewis DR, Chen HS, Feuer EJ, Cronin KA (eds). : SEER Cancer Statistics Review, 1975-2014. In., posted to the SEER web site, April 2017 edn. posted to the SEER web site, April 2017; based on November 2016 SEER data submission.
[13] Vriesema J, Poucki M, Kiemeney L, Witjes J. Patient opinion of urinary tests versus flexible urethrocystoscopy in follow-up examination for superficial bladder cancer: a utility analysis. Urology. 2000;56(5):793-7.

[14] Yossepowitch O, Herr HW, Donat SM: Use of urinary biomarkers for bladder cancer surveillance: patient perspectives. The Journal of Urology. 2007;177(4):1277-82.

[15] Langa KM, Larson EB, Wallace RB, Fendrick AM, Foster NL, Kabeto MU, Weir DR, Willis RJ, Herzog AR: Outof-pocket health care expenditures among older Americans with dementia. Alzheimer Dis Assoc Disord. 2004;18(2): 90-8.

[16] Simon M, Bosset P-O, Rouanne M, Benhamou S, Radulescu C, Molinié V, Neuzillet Y, Paoletti X, Lebret T: Multiple recurrences and risk of disease progression in patients with primary low-grade (TaG1) non-muscle-invasive bladder cancer and with low and intermediate EORTC-risk score. PloS one. 2019;14(2):e0211721.

[17] Torbit LA, Albiani JJ, Crangle CJ, Latini DM, Hart TL: Fear of recurrence: the importance of self-efficacy and satisfaction with care in gay men with prostate cancer. Psychooncology. 2015;24(6):691-8. 


\section{APPENDIX}

\section{Patient Satisfaction Questionnaire}

To be administered after the cystoscopy procedure.

1. Discomfortassociated with the procedure, please circle one:

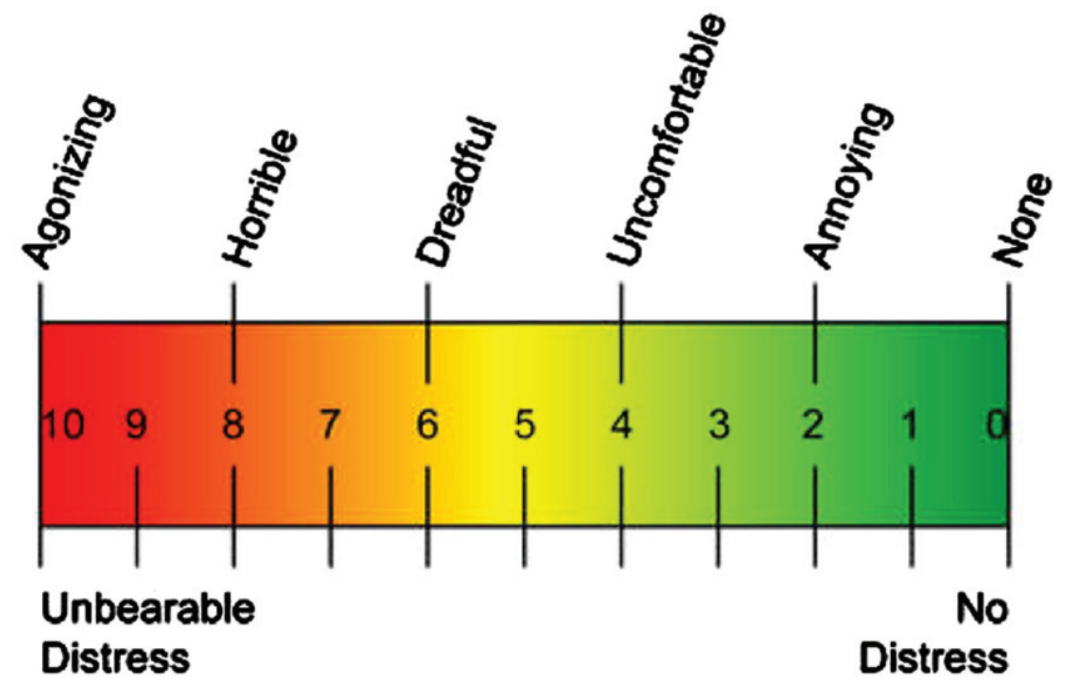

2. Bother associated with the procedure, please circle any that apply or provide your own explanation.

a. I feel better after this procedure because I feel that I'm taking good care of myself.

b. I am frustrated by this procedure and feel that it is performed too often and is not necessary.

c. Other, please explain: 


\section{Patient-reported Cost Assessment}

Time

Did you take time off of work for this procedure?

$\square$ Yes $\square$ No

If YES, how much time? (please indicate in hours):

Did someone accompanying you take time off of work for this procedure?

口 Yes $\mathrm{No}$

If YES, how much time? (please indicate in hours):

Transportation

How did you get to this appointment? (select all that apply):

口 Drove

Took public transportation, such as a bus

r Took a cab

口 Walked

Other, explain:

If you drove, what is the total mileage (round trip) driven for this appointment: Miles

What was your total (round trip) travel time: Hour(s)____ Minutes

\section{Moner}

How much money do you estimate this appointment cost you personally? (Assuming insurance covers what they are supposed to cover)

Any other expenses or time that we should account for because of this appointment that we missed from the above questions? 